\title{
Particular Solutions of the Equation of Conduction of Heat in One Dimension.
}

\author{
By Marion C. Gray.
}

(Received 18th November 1924. Read 16th Januury 1925.)

$\$ 1$. The problem of the conduction of heat in one dimension is usually concerned with the propagation of a thermal disturbance along a bar or rod of uniform cross section. The solution of the problem is required for a given initial distribution of temperature, and given boundary values, usually at each end of the rod. In most cases this solution is found by assuming a series solution and then proving that the series satisfies the equation of the disturbance as well as all the assigned conditions. Other methods, for example the contour integral method developed by Carsiaw, ${ }^{*}$ also introduce this arbitrary element of choice in choosing the integrand and the contour of integration. The object of the present paper is to develop the application of Heaviside's Operational method to the solution of the problem, and to show that it leads in all cases to solutions equivalent to the known forms, although initially no assumptions are made regarding the nature of the solution.

$\$ 2$. Heaviside's $\dagger$ formula, together with the allied ones of Bromwich $\ddagger$ and Carson $§$ may be briefly stated. The equation is obtained in the symbolic form

$$
v=\frac{Y(p)}{Z(p)} v_{0}
$$

where $Y$ and $Z$ are functions of the differential operator $p=\frac{d}{d t}$, $v_{0}$ is constant, and the initial instant is so chosen that at $t=0$ the disturbance is zero. Then the solution of the equation is

$$
v=v_{0}\left[\frac{Y(0)}{Z(0)}+\sum_{m} \frac{Y\left(p_{m}\right)}{p_{n} Z\left(p_{m}\right)} e^{p_{m}}\right]
$$

* H. S. Carslaw : Mathematical Theory of the Conduction of Heat in Solids. Ch. XI.

† Heavision: Electromagnetic Theory, Vol. II., Chapter V.

† Bromwich : Phil. Mag., London, (Ser. 6), 37, p. 407, 1918.

§ Carson: Physical Review, X., 2, 1917. 
where $p_{m}$ is a simple root of the algebraic equation $Z(p)=0$, and the summation extends over all such roots.

Bromwich's symbolic equation is

$$
v=\frac{Y(p)}{Z(p)} G t,
$$

where $G$ is a constant, and the solution he obtains is

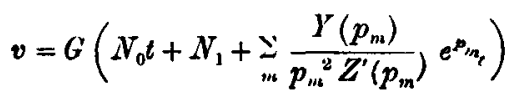

when $N_{0} N_{1}$ are determined by the equation

$$
\frac{Y(p)}{Z(p)}=N_{0}+N_{1} p+N_{2} p^{2}+\ldots .
$$

Carson gives a formula for the solution of the equation

$$
v=E \frac{Y(p)}{Z(p)} \epsilon^{a t}
$$

namely,

$$
v=E\left[\frac{Y(\alpha)}{Z(\alpha)} e^{a t}-\Sigma \frac{Y\left(p_{m}\right)}{\left(\alpha-p_{m}\right) Z^{\prime}\left(p_{m}\right)} e^{p_{m}}\right] .
$$

\$3. This formula of Carson's admits of a generalisation which will prove useful. Consider the symbolic equation

$$
u=\frac{Y(p)}{Z(p)} \phi(t),
$$

where $\phi(\ell)$ is such that it can be expanded as a Fourier integral

$$
\begin{aligned}
\phi(t) & =\frac{2}{\pi} \int_{0}^{\infty} \int_{-\infty}^{\infty} \cos u(\lambda-t) \phi(\lambda) d \lambda d u \\
& =\frac{1}{\pi} \int_{0}^{\infty} \int_{-\infty}^{\infty}\left\{e^{i u(\lambda-t)}+e^{-i u(\lambda-t)}\right\} \phi(\lambda) d \lambda d u
\end{aligned}
$$

Then $u=\frac{1}{\pi} \int_{0}^{\infty} \int_{-\infty}^{\infty} e^{i u \lambda} \phi(\lambda) \frac{Y(p)}{Z(p)} e^{-i u t} d \lambda d u$

$$
+\frac{1}{\pi} \int_{0}^{\infty} \int_{-\infty}^{\infty} e^{-i u \lambda} \phi(\lambda) \frac{Y(p)}{Z(p)} e^{i u t} d \lambda d u
$$


Write $u_{1}=\frac{1}{\pi} \int_{0}^{\infty} \int_{-\infty}^{\infty} e^{i u \lambda} \phi(\lambda) \frac{Y(p)}{Z(p)} e^{-i u t} d \lambda d u$.

Then by Carson's formula,

$$
\begin{aligned}
& u_{1}=\frac{1}{\pi} \int_{0}^{\infty} d u \int_{-\infty}^{\infty} d \lambda e^{i u \lambda} \phi(\lambda)\left\{\frac{Y(-i u)}{Z(-i u)} e^{-i u t}-\underset{m\left(-i u-p_{m}\right)}{Y} Z^{\prime}\left(p_{m}\right) e^{p_{m^{t}}}\right\} \\
& =\frac{1}{\pi} \int_{-\infty}^{0} d u \int_{-\infty}^{\infty} d \lambda e^{-i u \lambda} \phi(\lambda)\left\{\frac{Y(i u)}{Z(i u)} e^{i u t}-\sum_{m} \frac{Y\left(p_{m}\right)}{\left(i u-p_{m}\right) Z^{\prime}\left(p_{m}\right)} e^{\nu^{\prime} m^{t}}\right\}
\end{aligned}
$$

and the solution of the equation

$$
\begin{aligned}
& u_{2}=\frac{1}{\pi} \int_{0}^{\infty} \int_{-\infty}^{\infty} e^{-i u \lambda} \phi(\lambda) \frac{Y(p)}{Z(p)} e^{i u l} d \lambda d u \\
& \text { is } \quad u_{u}=\frac{1}{\pi} \int_{0}^{\infty} d u \int_{-\infty}^{\infty} d \lambda e^{-i u \lambda} \phi(\lambda)\left\{\frac{Y(i u)}{Z(i u)} e^{i u t}\right. \\
& -\underbrace{}_{m} \frac{Y\left(p_{m}\right)}{\left(i u-p_{m}\right) Z^{\prime}\left(p_{m}\right)} e^{p_{m^{t}}}\} \\
& \therefore u=u_{1}+u_{2}=\frac{1}{\pi} \int_{-\infty}^{\infty} d u \int_{-\infty}^{\infty} d \lambda e^{-i u \lambda} \phi(\lambda)\left[\frac{Y(i u)}{Z(i u)} e^{i u l}\right. \\
& \left.-\underset{m}{\searrow} \frac{Y\left(p_{m}\right)}{\left(i u-p_{m}\right) Z^{\prime}\left(p_{m}\right)} e^{p_{m^{t}}}\right] \\
& =\frac{1}{\pi} \int_{-\infty}^{\infty} d u \frac{Y(i u)}{Z(i u .)} e^{i u t} \int_{-\infty}^{\infty} d \lambda e^{-i u \lambda} \phi(\lambda) \\
& -\frac{1}{\pi} \sum_{m} \frac{Y\left(p_{m}\right)}{Z Z^{\prime}\left(p_{m}\right)} e^{p_{m} t} \int_{-\infty}^{\infty} \frac{d u}{i u-p_{m}} \int_{\infty}^{\infty} e^{-i u \lambda} \phi(\lambda) d \lambda \\
& =\frac{1}{\pi} \int_{-\infty}^{\infty} d u \frac{Y(i u)}{Z(i u)} e^{i u t} \int_{-\infty}^{\infty} d \lambda e^{-i u \lambda} \phi(\lambda) \\
& -2 \underset{m}{\Sigma} \frac{Y\left(p_{m}\right)}{2 Z\left(p_{m}\right)} e^{p^{\prime}} \int_{-\infty}^{\infty} e^{-\lambda p_{m} \phi(\lambda) d \lambda .}
\end{aligned}
$$

$\S 4$. We shall consider now the various problems in the conduction of heat in a finite rod as enunciated by Carslaw.* The first is: Finite Rod : ends at zero Temperature. Initial Temperature $f(x)$.

* Cargraw : Loc. cit., Chapter IV. 
No Radiation at the Surface. Analytically then the problem is the solution of the equations

$$
\begin{aligned}
& \frac{\partial v}{\partial t}=\kappa \cdot \frac{\partial^{2} v}{\partial x^{2}} \quad(0<x<l) \ldots \ldots \\
& v=0 \text { when } x=0 \text { and } x=l \\
& v=f(x) \text { when } t=0 \ldots \ldots \ldots
\end{aligned}
$$

It is assumed that $f(x)$ can be expanded as a Fourier sine series

$$
f(x) \sum a_{n} \sin \frac{n \pi x}{l}
$$

To satisfy the condition that the disturbance is zero at the initial instant write

$$
v=u+f(x)=u+\sum a_{n} \sin \frac{n \pi x}{l} .
$$

The differential equation becomes

$$
\frac{\partial u}{\partial t}=\kappa \frac{\partial^{2} u}{\partial x^{2}}-\kappa \Sigma a_{n} \frac{n^{2} \pi^{2}}{l^{2}} \sin \frac{n \pi x}{l}
$$

or, symbolically

$$
\kappa \frac{\hat{\sigma}^{2} u}{\partial x^{2}}-p u=\kappa \Sigma a_{n} \frac{n^{2} \pi^{2}}{l^{2}} \sin \frac{n \pi x}{l} .
$$

The solution of this equation, regarding $p$ as an algebraic constant, is

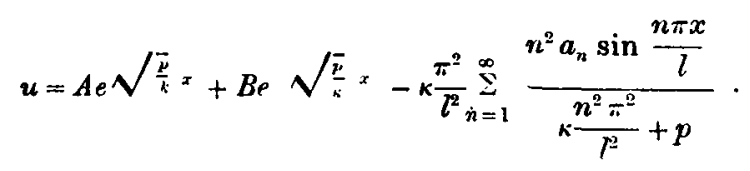

Now apply the conditions $(4 b)$ which give $u=0$ where $x=0$ and $x=l$.

$$
\begin{aligned}
& \therefore \quad 0=A+B \text {. } \\
& \therefore \quad 0=A e^{\sqrt{p}} \iota+B e^{-\sqrt{\frac{\mu}{\alpha}} l} \\
& \therefore A=B=0 \text {. } \\
& \therefore u=-\kappa \frac{\pi^{2}}{l^{2}} \stackrel{\infty}{n=1}_{\kappa}^{\infty} \frac{n^{2} a_{n} \sin \frac{n \pi x}{l}}{\kappa \frac{n^{2} \pi^{2}}{l^{2}}+p .} .
\end{aligned}
$$

The zeros of each denominator are different, and therefore each term can be considered separately. 
Consider

$$
u_{n}=-\frac{\kappa \frac{n^{2} \pi^{2}}{l^{2}} a_{n} \sin \frac{n \pi x}{l}}{\kappa \frac{n^{2} \pi^{2}}{l^{2}}+p} .
$$

This is in form 1 , with $Y(p)$ constant, $Z(p)=\kappa \frac{n^{2} \pi^{2}}{l^{2}}+p$.

Oniy root of $Z(p)=0$ is $p=-\kappa \frac{n^{2} \pi^{2}}{l^{2}}$.

For this root $\frac{Y(p)}{p Z^{\prime}(p)}=a_{n} \sin \frac{n \pi x}{l}$.

$\therefore$ Solution of the equation is

$$
u_{n}=-a_{n} \sin \frac{n \pi x}{l}+a_{n} \sin \frac{n \pi x}{l} e^{-\frac{n^{2} \pi^{2}}{l} l^{2}} t,
$$

with similar expressions for $u_{1} u_{2} \ldots \ldots \ldots \ldots$

$$
\begin{aligned}
& \therefore \quad u=-\sum a_{n} \sin \frac{n \pi x}{l}+\sum a_{n} \sin \frac{n \pi x}{l} e^{-\kappa \frac{n^{3} \pi^{2}}{l} l} \\
& \therefore \quad v=\sum_{n=1}^{\infty} a_{n} \sin \frac{n \pi x}{l} e^{-\kappa \frac{n=2}{l}=}
\end{aligned}
$$

which is the usual solution of the problem.

§5. Finite Rod. Ends at Fixed Temperatures. Initial Temperature $f(x)$. No Radiation.

The equations to be satisfied are

$$
\begin{aligned}
& \frac{\partial v}{\partial t}=\kappa \frac{\partial^{2} v}{\partial x^{2}}(0<x<l) \\
& v=v_{1} \text { when } x=0 \\
& \left.v=v_{2} \text { when } x=l\right\} \\
& v=f(x)=\Sigma a_{n} \sin \frac{n \pi x}{l} \text { when } t=0
\end{aligned}
$$

Again write $v=u+f(x)$ and consider only the $\boldsymbol{n}^{\text {th }}$ term of the series for $f(x)$.

Then $\left(\kappa D^{2}-p\right) u_{n}=\kappa a_{n} \frac{n^{2} \pi^{2}}{l^{2}} \sin \frac{n \pi x}{l}$

$$
\therefore \quad u_{n}=A e \sqrt{\frac{p}{\alpha} x}+B e \sqrt{\frac{p}{\alpha}} \times-\frac{\kappa a_{n} \frac{n^{2} \pi^{2}}{l^{2}} \sin \frac{n \pi x}{l}}{\frac{\kappa n^{2} \pi^{2}}{l^{2}}+p}
$$


Substitute from $(5 b)$, so that

$$
\begin{aligned}
& v_{i}=A+B \\
& v_{2}=A e \sqrt{\frac{\bar{p}}{\alpha}} l+B t^{-} \sqrt{\frac{\bar{p}}{\alpha}} l \\
& \therefore \quad A=\frac{v_{2}-v_{1} e^{-} \sqrt{\frac{\bar{p}_{k}}{x} l}}{2 \sinh \sqrt{\frac{\bar{p}_{x}}{x} l}} \\
& B=\frac{v_{1} e \sqrt{\frac{\bar{z}}{\alpha} l}-v_{2}}{2 \sinh \sqrt{\frac{\bar{z}_{x}}{\alpha} l}} \\
& \therefore \quad u_{n}=\frac{v_{2} \sinh \sqrt{\frac{p}{\kappa}} x+v_{1} \sinh \sqrt{\frac{p}{\kappa}}(l-x)}{\sinh \sqrt{\frac{p}{\kappa}} l}-\frac{\kappa a_{n} \frac{n^{2} \pi^{2}}{l^{2}} \sin \frac{n \pi x}{l}}{\kappa \frac{n^{2}}{l^{2}}+p} \\
& Z(p)=\sinh \sqrt{\frac{p}{\kappa}} l\left(\kappa \frac{n^{2} \pi^{2}}{l^{2}}+p\right)
\end{aligned}
$$

with poles at $\sqrt{\frac{\bar{p}}{\kappa} l}=i r \pi \quad(r=0,1,2, \ldots \ldots)$

$$
\begin{aligned}
& \therefore \quad p_{r}=-\frac{\kappa r^{2} \pi^{2}}{l^{2}} \\
& p_{r} Z^{\prime}\left(p_{r}\right)=p_{r}\left(\kappa \frac{n^{2} \pi^{2}}{l^{2}}+p_{r}\right) \frac{l}{2 \sqrt{p_{r} \kappa}} \cosh \sqrt{\frac{p_{r}}{\kappa}} l+p_{r} \sinh \sqrt{\frac{p_{r}}{\kappa} l} \\
& =(-1) r \frac{i r \pi}{2} \kappa \frac{\left(n^{2}-r^{2}\right) \pi^{2}}{l^{2}} \\
& Y\left(p_{r}\right)=\frac{\kappa\left(n^{2}-r^{2}\right) \pi^{2}}{l^{2}}\left(v_{2} \sinh \frac{i r \pi x}{l}+v_{1} \sinh \frac{i r \pi(l-x)}{l}\right) \\
& =i \kappa \frac{\left(n^{2}-r^{2}\right) \pi^{2}}{l^{2}}\left(v_{2}+(-1)^{r-1} v_{1}\right) \sin \frac{r \pi x}{l} \text {. } \\
& \therefore \frac{Y\left(p_{r}\right)}{p_{r} Z\left(p_{r}\right)}=\frac{2}{r \pi}\left((-1)^{r} v_{2}-v_{1}\right) \sin \frac{r \pi x}{l} \text {. } \\
& \text { For } p=-\kappa \frac{n^{2} \pi^{2}}{l^{2}} \text { we have the additional term } \\
& \frac{-\kappa a_{n} \frac{n^{2} \pi^{2}}{l^{2}} \sin \frac{n \pi x}{l}}{-\kappa \frac{n^{2} \pi^{2}}{l^{2}}}=a_{n} \sin \frac{n \pi x}{l}
\end{aligned}
$$


We have still to determine $\frac{Y(0)}{Z(0)}$.

$$
\begin{aligned}
& Y(p)=\left(\kappa \frac{n^{2} \pi^{2}}{l^{2}}+p\right)\left\{v_{2}\left[\sqrt{\frac{p}{\kappa}} x+\frac{1}{3 !}\left(\sqrt{\frac{p}{\kappa}} x\right)^{3}+\ldots\right]\right. \\
& \left.+v_{1}\left[\sqrt{\frac{n}{\kappa}}(l-+x) \frac{1}{3 !}\left(\sqrt{\frac{p}{\kappa}} \overline{l-x}\right)^{3}+\ldots\right]\right\} \\
& \text {.. } \kappa a_{n} \frac{n^{2} \pi^{2}}{l^{2}} \sin \frac{n \pi x}{l}\left\{\sqrt{\frac{p}{\kappa}} l+\frac{1}{3 !}\left(\sqrt{\frac{p}{\kappa}} l\right)^{3}+\ldots\right\} \\
& =\kappa \frac{n^{2} \pi^{n}}{l^{2}}\left(x v_{2}+\overline{l-x} v_{1}-l x_{n} \sin \frac{n \pi x}{l}\right) \sqrt{\frac{p}{\kappa}}+O\left(\nu^{\prime \prime / *}\right) \text {. } \\
& Z(p)=\left(\kappa \frac{n^{\prime \prime} \pi^{\prime \prime}}{l^{2}}+p\right)\left(\sqrt{\frac{p}{\kappa} l} l+\frac{1}{3 !}\left(\sqrt{\frac{p}{\kappa}} l\right)^{!}+\ldots\right) \\
& =\kappa \frac{n^{\prime 2} \pi^{2}}{l^{2}} l \sqrt{\frac{p}{\kappa}}+O(p) \text {. } \\
& \therefore \frac{Y(0)}{Z(0)}=\frac{x v_{2}+(l-x) v_{1}-l a_{n} \sin \frac{n \pi x}{t}}{l} \\
& =v_{1}+\frac{\left(v_{2}-v_{1}\right) x}{l}-a_{n} \sin \frac{n \pi x}{l} \text {. }
\end{aligned}
$$

Collecting terms, the solution is given by

$\therefore$ Finally,

$$
\begin{aligned}
& u_{n}=v_{1}+\frac{\left(v_{n}-v_{1}\right) x}{l}-a_{n} \sin \frac{n \pi x}{l}+a_{n} \sin \frac{n \pi x}{l} e^{-\kappa \frac{n=\pi}{l^{2}} l} \\
& +\frac{2}{\pi} \sum_{r=0}^{n} \frac{v \cos r \pi-v_{1}}{r} \sin \frac{r \pi x}{l} e^{-r^{r=} r^{*} t}
\end{aligned}
$$

$$
\begin{aligned}
& v=v_{1}+\begin{array}{c}
\left(v_{2}-v_{1}\right) x \\
l
\end{array}+\underbrace{\infty}_{n=1} a_{n} \sin \frac{n \pi x}{l} e^{-\kappa \frac{n_{i}^{n} z^{2}}{l^{\prime \prime}} t} \\
& +\frac{2}{\pi} \underbrace{\infty}_{r=0} \frac{v_{2} \cos r \pi-v_{1}}{r} \sin \frac{r \pi x}{l} e^{-\kappa \frac{i \cdot \pi^{*}}{l^{*}}-t}
\end{aligned}
$$

$\$ 6$. Finite Rod. Ends at Temperatures $\phi_{1}(t)$ and $\phi_{:}(t)$. Initial Temperature $f(x)$. No radiation. The equations to be satisfied are

$$
\begin{aligned}
& \frac{\partial v}{\partial t}-\kappa \frac{\hat{v}^{2} v}{\hat{\imath} x^{2}} \\
& v=\phi_{1}(t) \text { when } x=0 \text { ) } \\
& \left.v=\phi_{2}(t) \text { when } x=l\right\} \\
& v=f(x)=\check{\Sigma} a_{n} \sin \frac{n \pi x}{l} \text { when } t=0
\end{aligned}
$$


57

W rite $v=u+f(x)$ and as before

$$
\begin{aligned}
& u=\frac{\operatorname{inh} \sqrt{\frac{p}{\kappa}} x}{\sinh \sqrt{\frac{p}{\kappa}},} \phi_{2}(t)+\frac{\sinh \sqrt{\frac{p}{\kappa}}(l-x)}{\sinh \sqrt{\frac{p}{\kappa}} l} \phi_{1}(t) \\
& \text { Consider first } u_{1}=\frac{-\sum_{n} \frac{\kappa a_{n} \frac{n^{2} \pi^{2}}{l^{2}} \sin \frac{n \pi x}{l}}{\kappa \frac{n^{2} \pi^{2}}{l^{2}}+p}}{\sinh \sqrt{\frac{p}{\kappa}} x} \phi_{2}(t),
\end{aligned}
$$

and apply the Formula (6).

$$
\begin{aligned}
& \therefore \quad u_{1}=\frac{1}{\pi} \int_{-\infty}^{\infty} d \alpha \frac{\sinh \sqrt{\frac{i \alpha}{\frac{i \alpha}{\alpha}} x}}{\sinh \sqrt{\sqrt{\frac{i \alpha}{\kappa}}},} e^{i \alpha t} \int_{-\infty}^{\infty} e^{-i \alpha \lambda} \phi_{2}(\lambda) d \lambda \\
& -2 \sum_{n=-\infty}^{\infty} \frac{i \sin \frac{n \pi x}{l}}{l^{2} \cos n \pi} 2 i n \pi \kappa e^{-\frac{n^{2} \pi^{2} u}{l^{2}} t} \int_{-\infty}^{\infty} e^{\frac{n^{2} \pi^{2} \kappa}{l^{2}} \lambda} \phi_{2}(\lambda) d \lambda . \\
& \text { But } \frac{\sinh \sqrt{\frac{i \alpha}{\kappa}} x}{\sinh \sqrt{\frac{i \alpha}{\kappa}} /}=2 \pi \kappa \sum_{n=1}^{\infty} \frac{n(-1)^{n+1}}{i \alpha l^{2}+n^{2} \pi^{2} \kappa} \sin \frac{n \pi x}{l}
\end{aligned}
$$

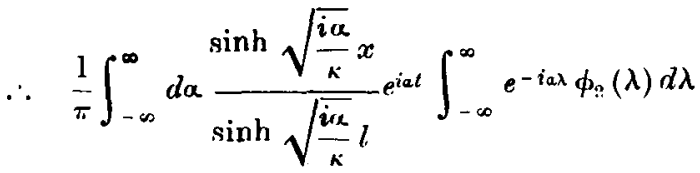

$$
\begin{aligned}
& =2 \kappa \sum_{n=0}^{\infty} n(-1)^{n+1} \sin \frac{n \pi x}{l} \int_{-\infty}^{\infty} e^{i a(t-\lambda)} \frac{d \alpha}{i \alpha l^{2}+n^{2} \pi^{2} \kappa} \int_{-\infty}^{\infty} \phi_{2}(\lambda) d \lambda \\
& =2 \kappa \sum_{n=0}^{\infty} n(-1)^{n+1} \sin \frac{n \pi x}{l} \int_{-\infty}^{\infty} \frac{2 \pi}{l^{3}} e^{-\frac{n^{2} \pi^{2} k_{l t}}{l^{2}}}-\lambda_{l} \phi_{2}(\lambda) d \lambda
\end{aligned}
$$

https://doi.org/10.1017/S0013091500036312 Published online by Cambridge University Press 


$$
\begin{aligned}
& \therefore \quad u_{1}=\frac{4 \pi \kappa}{l^{2}} \sum_{n=0}^{\infty} n(-1)^{n+1} \sin \frac{n \pi x}{l} e^{-\frac{n \pi^{2}=x}{l^{2}} t} \int_{-\infty}^{\infty} e^{\frac{n^{2} \pi^{2} x}{l=} \lambda} \phi_{2}(\lambda) d \lambda \\
& +\frac{4 \pi \kappa}{l^{2}} \sum_{n=-\infty}^{\infty} n(-1)^{n} \sin \frac{n \pi x}{l} e^{-\frac{n^{2} \pi^{2} k}{l^{2}}} t \int_{-\infty}^{\infty} e^{\frac{n^{2} \pi^{2} k}{l=} \lambda} \phi_{2}(\lambda) d \lambda \\
& =\frac{2 \pi \kappa}{l^{2}} \sum_{n=-\infty}^{\infty} n(-1)^{n+1} e^{-\frac{n^{2} x^{2} \kappa}{l^{2}} l} \sin \frac{n \pi x}{l} \int_{-\infty}^{\infty} e^{\frac{n^{2} \Sigma^{2} k}{l^{2}} \lambda} \phi_{2}(\lambda) d \lambda \text {. } \\
& \text { Similarly if we write } u_{2}=\frac{\sinh \sqrt{\frac{p}{\kappa}}(l-x)}{\sinh \sqrt{\frac{p}{\kappa}} l} \phi_{1}(t)
\end{aligned}
$$

we have $\quad u_{2}=\frac{2 \pi k}{l^{2}} \sum_{n=-\infty}^{\infty} n \sin \frac{n \pi x}{l} e^{-\frac{n^{2} \pi^{2} k}{l^{2}} t} \int_{-\infty}^{\infty} e^{\frac{n^{2} \pi^{2} k}{l^{2}}{ }^{2}} \phi_{l}(\lambda) d \lambda$.

$$
\text { Also if } u_{3}=-\Sigma \frac{\kappa a_{n} \frac{n^{2} \pi^{8}}{l^{2}} \sin \frac{n \pi x}{l}}{\kappa \frac{n^{2} \pi^{2}}{l^{2}}+p}
$$

the solution is, from $\$ 4$

$$
u_{3}=-\sum_{n=1}^{\infty} a_{n} \sin \frac{n \pi x}{l}+\sum_{n=1}^{\infty} a_{n} \sin \frac{n \pi x}{l} \cdot-\frac{k n^{3} n^{2}}{l^{2}} t
$$

Finally $v=u_{1}+u_{2}+u_{3}+f(x)$

$$
\begin{aligned}
& =\sum_{n=1}^{\infty} a_{n} \sin \frac{n \pi x}{l} e^{-x \frac{n=\pi^{2}}{l^{2}} t} \\
& +\frac{2 \pi \kappa}{l^{2}} \sum_{n=-\infty}^{\infty} n \sin \frac{n \pi x}{l} e^{-\kappa \frac{n^{2} \pi^{2}}{l^{2}}} t \int_{-\infty}^{\infty} e^{\frac{n^{2} n^{2} n}{l^{2}} \lambda}\left\{\phi_{1}(\lambda)-(-1)^{n} \phi_{2}(\lambda)\right\} d \lambda . \\
& =\frac{2}{l} \sum_{n=1}^{\infty} e^{-k \frac{n^{2} \pi^{2} l}{l^{2}}} \sin \frac{n \pi x}{l}\left[\int_{0}^{l} f\left(x^{\prime}\right) \sin \frac{n \pi x^{\prime}}{l} d x^{\prime}\right. \\
& \left.+\frac{n \pi x}{l} \int_{0}^{t} e^{\frac{n^{2} \pi^{2} x}{l^{2}} \lambda}\left\{\phi_{1}(\lambda)-(-1)^{n} \phi_{2}(\lambda)\right\} d \lambda\right]
\end{aligned}
$$

since $\phi(t)$ must vanish for $t<0$, and the values of $\phi$ for times later than the instant $t$ under connideration need not be considered. 
§7. Finite Rod. Radiation at Ends into a Medium at Zero Temperature. Initial Temperature $f(x)$. No Radiation at the Surface.

The differential equations are

$$
\left.\begin{array}{c}
\frac{\partial v}{\partial t}=\kappa \frac{\partial^{2} v}{\partial x^{2}} \ldots \ldots \ldots \ldots \ldots \ldots \ldots \ldots \ldots \ldots \ldots \ldots \ldots \ldots \ldots \ldots \ldots \ldots \ldots \ldots \\
-\frac{\partial v}{\partial x}+h v=0 \text { at } x=0 \\
\frac{\partial v}{\hat{\imath} x}+h v=0 \text { at } x=l
\end{array}\right\} \ldots
$$

Put $v=u+f(x)$, and wo have as before,

$$
u=\Lambda e \sqrt{\frac{\bar{p}}{x} x}+B e^{-} \sqrt{\frac{\bar{p}}{x}} x-\Sigma \frac{\kappa n^{2} \pi^{2} a_{n}}{l^{2}} \frac{\sin \frac{n \pi x}{l}}{\kappa \frac{n^{2} \pi^{2}}{l^{2}}+p}
$$

Write $\sqrt{\frac{p}{\kappa}}=s$

$$
\therefore \quad u=A e^{2 x}+B e^{-t x}-\Sigma \frac{n^{2} \pi^{2} a_{n}}{n^{2} \pi^{2}+l^{2} s^{2}} \sin \frac{n \pi x}{l} .
$$

Now $-\frac{\partial v}{\partial x}+h v=-\frac{\partial u}{\partial x}+h u-\Sigma \frac{n \pi a_{n}}{l} \cos \frac{n \pi x}{l}+h \Sigma a_{n} \sin \frac{n \pi x}{l}$.

At $x=0,-\frac{\partial v}{\partial x}+h v=-\frac{\partial u}{\partial x}+h u-\Sigma \frac{n \pi a_{n}}{7}$

$$
\begin{aligned}
\therefore \quad 0= & -A s+B s+\sum \frac{n \pi}{l} \frac{n^{2} \pi^{2} a_{n}}{n^{2} \pi^{2}+l^{2} s^{2}} \\
& \quad+h A+h B-\sum \frac{n \pi a_{n}}{l} \\
= & A(h-s)+B(h+s)-\sum \frac{n \pi a_{n} l s^{2}}{n^{2} \pi^{2}+l^{2} s^{2}} \ldots \ldots\left(7, b_{1}\right)
\end{aligned}
$$

At $x=l . \quad \frac{\partial v}{\partial x}+h v=\frac{\partial u}{\partial x}+h u+\Sigma \frac{n \pi a_{n} \cos n \pi}{l}$ 


$$
\begin{aligned}
\therefore \quad 0=s A e^{a l}-s B e^{-d}- & \leq \frac{n^{3} \pi^{3}}{l} \frac{a_{n} \cos n \pi}{n^{2} \pi^{2}+l^{2} s^{2}} \\
& +h A e^{2 l}+h B e^{-y}+\leq \frac{n \pi a_{n} \cos n \pi}{l .} \\
=(h+s) e^{n} A+ & (h-s) e^{-y} B \\
& +\sum \frac{n \pi a_{n} l s^{2}}{n^{2} \pi^{2}+l^{2} s^{2}} \cos n \pi \ldots \ldots .\left(7, b_{2}\right)
\end{aligned}
$$

Solving ( $\left.7 b_{1}\right)$ and $\left(7 b_{3}\right)$ for $A$ and $B$ we have the equation in $u$.

$$
\begin{array}{r}
u=\sum_{n} \frac{n \pi a_{n} s^{2} l\left\{\left[(h-s) e^{-s}+(h+s) \cos n \pi\right] e^{s x}-\left[(h+s) e^{a l}+(h-s) \cos n \pi\right] e^{-s r}\right\}}{\left(n^{2} \pi^{2}+l^{2} s^{2}\right)\left\{(h-s)^{2} e^{-s l}-(h+s)^{2} e^{s t}\right\}} \\
-\sum_{n}^{\sum} \frac{n^{2} \pi^{2} a_{n} \sin \frac{n \pi x}{l}}{n^{2} \pi^{2}+l^{2} s^{2}} .
\end{array}
$$

$$
\text { Consider } u_{1}=-\frac{n^{2} \pi^{2} a_{n} \sin \frac{n \pi x}{l}}{n^{2} \pi^{2}+l^{2} s^{2}}
$$

with pole at $s^{2}=-\frac{n^{2} \pi^{2}}{l^{2}} . \quad \frac{1}{2} s \frac{d Z}{d s}=l^{2} s^{2}=-n^{2} \pi^{2}$.

$$
\begin{gathered}
\frac{2 Y(s)}{s Z^{\prime}(s)}=a_{n} \sin \frac{n \pi x}{l} \\
\therefore \quad u_{1}=-a_{n} \sin \frac{n \pi x}{l}+a_{n} \sin \frac{n \pi x}{l} e^{-\kappa} \frac{n^{2} \pi^{2}}{l^{2}} l
\end{gathered}
$$

Now let $u_{2}=$

$$
\begin{gathered}
\frac{n \pi a_{n} s^{2} l\left[\left\{(h-s) e^{-s l}+(h+s) \cos n \pi\right\} e^{s x}-\left\{(h+s) e^{2 l}+(h-s) \cos n \pi\right\} e^{-s z}\right]}{\left(n^{2} \pi^{2}+l^{2} s^{2}\right)\left\{(h-s)^{2} e^{-s l}-(h+s)^{2} e^{2 l}\right\}} \\
Z(s)=\left(n^{2} \pi^{2}+l^{2} s^{2}\right)\left\{(h-s)^{2} e^{-s}-(h+s)^{2} e^{2 l}\right\}
\end{gathered}
$$

Zeros of $Z(s)$ are at $s^{2}=-\frac{n^{2} x^{2}}{l}$ and $(h-s)^{2} e^{-s}=(h+s)^{2} e^{2 l}$.

$$
\begin{gathered}
\text { i.e. } \quad e^{s l}=\frac{h-s}{h+s} \\
\frac{1}{2} s Z^{\prime}(s)=l^{2} s^{2}\left\{(h-s)^{2} e^{-s l}-(h+s)^{2} e^{s}\right\}+\frac{1}{2} s\left(n^{2} \pi^{2}+l^{2} s^{2}\right) \\
{\left[\left\{-2(h-s)-l(h-s)^{2}\right\} e^{-s l}-\left\{2(h+s)+l(h+s)^{2}\right\} e^{s l}\right]} \\
=l^{2} s^{2}\left\{(h-s)^{2} e^{-s}-(h+s)^{2} e^{l}\right\}-\frac{1}{2} s\left(n^{2} \pi^{2}+l^{2} s^{2}\right) \\
\left\{(2+l h-l s)(h-s) e^{-l}+(2+l h+l s)(h+s) e^{2 l}\right\} .
\end{gathered}
$$


$s^{2}=-\frac{n^{2} \pi^{2}}{l^{2}} \quad$ Then $\frac{1}{2} s Z^{\prime}(s)=-u^{2} \pi^{2}\left\{(h-s)^{2}+(h+s)^{2}\right\} \cos n \pi$

$$
=\frac{ \pm i h n^{2} \pi^{3}}{l} \cos u \pi \text {. }
$$

$$
\begin{aligned}
& Y(s)=4 \pi a_{n} s^{2} l\left[\left\{(h-s) e^{-s l}+(h+s) \cos n \pi\right\} e^{s x}\right. \\
& -\left\{(h+s) e^{\mu t}+(h-s) \cos n \pi ; e^{-s x}\right\} . \\
& \therefore \quad y\left(\frac{i n \pi}{l}\right)=-\frac{n^{3} \pi^{3} a_{n}}{l}\left[2 h \cos n \pi e^{\frac{i n \pi x}{l}}-2 h \cos n \pi e^{\frac{-i n \pi x}{l}}\right] \\
& =-\frac{4 i h n^{3} \pi^{3} a_{n} \cos n \pi}{l} \sin \frac{n \pi x}{l} . \\
& \therefore \quad \frac{2 Y\left(\frac{i n \pi}{l}\right)}{\frac{i n \pi}{l} Z^{\prime}\left(\frac{i n \pi}{l}\right)}=-a_{n} \sin \frac{n \pi x}{l} .
\end{aligned}
$$

Also $\frac{Y(0)}{Z(0)}=0$; therefore term contributed to $u_{2}$ by this root of $Z(s)=0$ cancels the corresponding term in $u_{1}$. Now consider the roots given by $e^{*}=\frac{h-s}{h+s,}$ or $e^{-s t}=\frac{h+s}{h-s}$.

$$
\begin{aligned}
& \text { Then } \frac{1}{2} s Z^{\prime}(s)=\frac{1}{2} s\left(n^{2} \pi^{2}+l^{2} s^{2}\right)\left\{(2+l h-l s)(h-s) e^{-s t}\right. \\
& \left.+(2+l h+l s)(h+8) e^{s l}\right\} \\
& =\frac{1}{2} s\left(n^{2} \pi^{2}+l^{2} s^{2}\right)\{(2+l h-l s)(h+s) \\
& +(2+l h+l s)(h-s)\} \\
& =s\left(n^{2} \pi^{2}+l^{2} s^{2}\right)\left(2 h+l h^{2}-l s^{2}\right) . \\
& Y(s)=n \pi a_{n} s^{2} l\left[(h+s)(1+\cos n \pi) e^{a x}\right. \\
& \left.-(h-8)(1+\cos n \pi) e^{-\alpha x}\right] \\
& =n \pi a_{n} s^{2} l(1+\cos n \pi)(h+8)\left(e^{a x}-e^{s(l-x)}\right) . \\
& \therefore \quad v=\sum_{n=1}^{\infty} \operatorname{S}_{S} n \pi a_{n} l(1+\cos n \pi) \frac{s(h+s)\left\{e^{a x}-e^{s(l-x)}\right\}}{\left(n^{2} \pi^{2}+l^{2} s^{2}\right)\left(2 h+l h^{2}-l s^{2}\right)} e^{x s=t}
\end{aligned}
$$

where the summation for $s$ is extended over all the roots of the transcendental equation

$$
e^{2}=\frac{h-8}{h+8}
$$


$\S 8$. The solution of the problem of $\S 7$ is usually given in the form

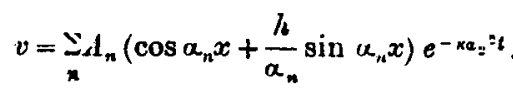

where $a_{n}$ is a root of the equation

$$
\tan u l=\frac{2 \alpha h}{a^{2}-h^{2}}
$$

and the summation extends over all such roots. Further $f^{\prime}(x)$ is expanded as a series

$$
f^{\prime}(x)=\Sigma A_{n}\left(\cos \alpha_{n} x+\frac{\hbar}{a_{n}} \sin \alpha_{n} x\right)
$$

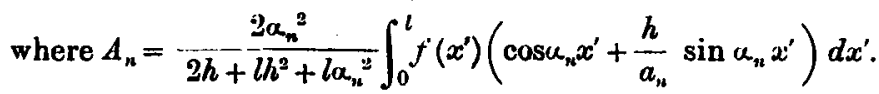

To show the identity of the two solutions, put $s=i \alpha$ in (7),

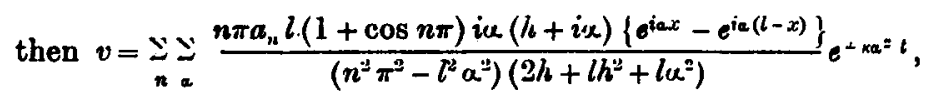

and the equation for $s$ becomes

$$
\begin{gathered}
e^{i \alpha l}=\frac{h-i \alpha}{h+i \alpha} \\
\therefore \quad \tan \alpha l=\frac{\Xi \alpha h}{\alpha^{2}-h^{2}} .
\end{gathered}
$$

$$
\begin{aligned}
& \text { Also } v=\sum_{n} \sum_{\kappa} \frac{n \pi a_{n} l(1+\cos n \pi) i \alpha}{\left(n^{3} \pi^{2}-l^{2} \alpha^{2}\right)\left(2 h+l h^{2}+l \alpha^{2}\right)} \\
& \left.\{h+i \alpha) e^{i \alpha x}-(h-i \alpha) e^{-i \alpha x}\right\} e^{-k a=l} \\
& =\sum \sum \sum \frac{n \pi a_{n} l(1+\cos n \pi) i \alpha}{\left(n^{2} \pi^{2}-l^{2} \alpha^{2}\right)\left(2 h+l h^{2}+l \alpha^{2}\right)} \\
& (2 i h \sin \alpha x+2 i \alpha \cos \alpha x) e^{-\kappa \alpha^{2} l} \\
& =\sum_{n a} \sum_{a}-\frac{2 n \pi a_{n} l(1+\cos n \pi) \alpha^{2}}{\left(n^{2} \pi^{2}-l^{2} \alpha^{2}\right)\left(2 h+l h^{2}+l \alpha^{2}\right)} \\
& \left(\cos \alpha x+\frac{h}{a} \sin \alpha x\right) e^{-k \alpha=1}
\end{aligned}
$$

In the equation for $A_{n}$ substitute the value of $f(x)$ given by equation (7c). 


$$
\begin{aligned}
& \text { Then } A_{n}=\frac{2 \alpha_{n}{ }^{2}}{2 h+l l^{2}+l \alpha_{n}^{2}} \int_{0}^{l}\left(\grave{l} a_{r} \sin \frac{r \pi x}{l}\right) \\
& \left(\cos \alpha_{n} x+\frac{h}{\alpha_{n}} \sin \alpha_{n} x\right) d x \\
& =-\frac{a_{n}^{2} a_{r}}{2 h+l h^{2}+l \alpha_{n}^{2}} \int_{0}^{l}\left\{\sin \left(\frac{r \pi}{l}+a_{n}\right) x+\sin \left(\frac{r \pi}{l}-a_{n}\right) x\right. \\
& +\frac{h}{a_{n}} \cos \left(\frac{r \pi}{l}-\alpha_{n}\right) x-\frac{h}{\alpha_{n}} \cos \left(\frac{r \pi}{l}+\alpha_{n}\right) x j d x \\
& =\sum_{r} \frac{\alpha_{n}{ }^{2} a_{r} l}{2 h+l h^{2}+l \alpha_{n}{ }^{2}} \frac{2 r \pi}{r^{2} \pi^{2}-\alpha_{n 2}{ }^{2} \lambda^{2}} \\
& \left\{1-\cos r \pi\left(\cos u_{n} l+\frac{h}{a_{n}} \sin a_{n} l\right)\right\} \\
& =\frac{2 \alpha_{n}^{2} a_{r} \ln \pi(1+\cos n \pi)}{r\left(2 h+l h^{2}+l a_{n}^{2}\right)\left(r^{2} \pi^{2}-\alpha_{n}^{2} l^{2}\right)} .
\end{aligned}
$$

Substituting this value of $A_{n}$ in (8) we have

$$
v=\underbrace{}_{r} \underset{a}{=} \frac{2 \alpha^{2} a_{r} r \pi l(1+\cos r \pi)}{\left(2 h+l h^{2}+l \alpha^{3}\right)\left(r^{2} \pi^{2}-\alpha^{2} l^{2}\right)}\left(\cos \alpha x+\frac{h}{\alpha} \sin \alpha x\right) e^{-\kappa \alpha^{2} t}
$$

which is identical with the form $\left(7^{\prime}\right)$. 